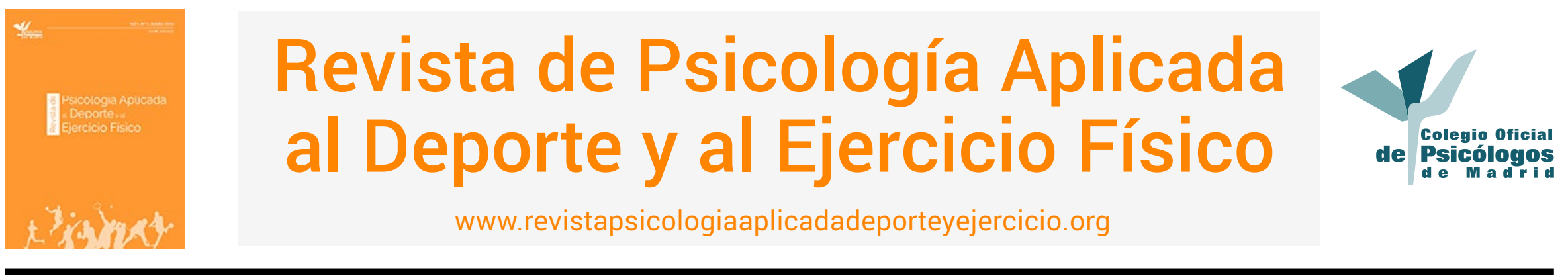

\title{
Establecimiento de objetivos en el currículum formativo de los futbolistas
}

\author{
Eduardo Morelló Tomás ${ }^{1}$, Bernardo Vert Boyer ${ }^{1}$ y Sergio Navarro Barquero² \\ 'Departamento de Psicología del Villarreal C.F., España, ${ }^{2}$ Departamento de Metodología del Villarreal C.F., España
}

RESUMEN: El Villarreal C.F dedica mucha atención a la formación de sus jóvenes jugadores tanto a nivel deportivo como a nivel académico y personal. Todo el proceso educativo se dirige a proponer al jugador entornos de aprendizaje que le permitan convertirse en un jugador autónomo (responsable de su proceso educativo, deportivo y personal), que entienda el juego (capaz de tomar decisiones analizando el entorno cambiante) y tenga valores para ser una persona íntegra y comprometida con la sociedad. En este artículo se presenta el proceso formativo seguido por los técnicos y jugadores para desarrollar un programa en establecimiento de objetivos como base para la mejora de la autonomía, entendimiento del juego y adquisición de valores. Los resultados de la valoración del mismo indican una buena satisfacción con el programa en general, la utilidad de la técnica y motivación para seguir aplicándola. Se concluye que la técnica en establecimiento de objetivos es eficaz para el desarrollo de personas y la mejora del rendimiento, liderada por el psicólogo para el beneficio de la institución, los entrenadores y jugadores.

PALABRAS CLAVES: Desarrollo personal, entrenamiento psicológico y metodología.

\section{Goal setting in football players curriculum}

ABSTRACT: Villarreal C.F devotes a lot of attention to training of its young players both at sports level and at the academic and personal levels. The whole educational process is aimed at proposing to the player learning environments that allow him to become an autonomous player (responsible for his educational, sporting and personal process) who understands the game (able to make decisions after analyzing the changing environment) and has values that allow him to become an upstanding person committed to society. This article details the training process followed by technicians and players to develop a goal setting program as a basis for improving autonomy, understanding of the game and acquisition of values in the players. The results of the evaluation suggest a good satisfaction with the overall program, the value of the technique, and motivation to continue to apply it. It is concluded that the goal setting technique is effective for personal development and improvement of performance when led by psychologists for the benefit of the institution, coaches and players.

KEYWORDS: Personal development, psychological training and methodology.

\section{Definir metas no currículo de formação dos jogadores}

RESUMO: O Villarreal C.F dedica muita atenção à formação dos seus jovens jogadores, tanto a nível desportivo como académico e pessoal. Todo o processo educativo se foca em propor ao jogador ambientes de aprendizagem que lhe permitam tornar-se um jogador autónomo (responsável pelo seu processo educativo, desportivo e pessoal), que entende o jogo (capaz de tomar decisões, analisando o ambiente em mudança) e que tem valores para ser uma pessoa íntegra e comprometida com a sociedade. Este artigo descreve o processo formativo seguido pelos técnicos e jogadores para desenvolver um programa de estabelecimento de metas como uma base para melhorar a autonomia, a compreensão do jogo e a aquisição de valores. Os resultados da avaliação indicam um bom nível

\footnotetext{
Eduardo Morelló Tomás, es psicólogo. Departamento de Psicología del Villarreal C.F.; Bernardo Vert Boyer, es psicólogo. Departamento de Psicología del Villarreal C.F.; Sergio Navarro Barquero, es entrenador de fútbol (UEFA PRO). Departamento de Metodología del Villarreal C.F. 
de satisfação com o programa em geral, a utilidade da técnica e motivação para continuar a aplicá-lo. Conclui-se que a técnica de definição de objetivos é eficaz para o desenvolvimento de pessoas e a melhoria do desempenho, liderada pelo psicólogo para benefício da instituição, dos treinadores e dos jogadores.

PALAVRAS-CHAVE: Desenvolvimento pessoal, treino psicológico e metodologia.

Artículo recibido: 08/01/2018 | Artículo aceptado: 05/03/2018

En el presente artículo se explica la experiencia profesional vivida del Departamento de Psicología del Deporte del Villarreal C.F. durante las temporadas 2014-2015 y 20152016. En este período de tiempo se desarrolló una intervención (explicada a continuación) que trató de definir y mejorar algunas de las cualidades que se pretendían desarrollar en los futbolistas (autonomía, visión de juego y entrenamiento en valores). Lo relevante en este caso es que la herramienta principal que se aplicó para dicho fin es la técnica motivacional en establecimiento de objetivos (Locke, Shaw, Saari y Lathan, 1981), ampliamente conocida y utilizada por los psicólogos en el ámbito del deporte (Weinberg y Gould, 2007). Al tratarse de una experiencia eminentemente aplicada, a continuación se describen los objetivos y procedimientos realizados de manera que el lector pueda entender la intención de cada acción realizada.

Son varios los artículos en España donde ya se enuncian las principales funciones de un Departamento de Psicología en un club de fútbol profesional (ver Morilla, Pérez, Gamito, Gómez y Valiente, 2003), así como los objetivos principales del fútbol base, como formar personas y jugadores que lleguen al fútbol profesional (ver García-Naveira y Jerez, 2012). Estos autores destacan en dichos artículos aspectos básicos coincidentes en nuestra organización, como:

- El jugador como proyecto: se trata de poner el foco en el jugador y desarrollarlo al máximo, integrado en el equipo y en el Club pero con una atención individualizada.

- La potencialidad del jugador: descubrir no solo lo que el jugador hace actualmente sino lo que puede llegar a hacer en el futuro. El jugador como deportista y persona, son un todo indivisible, y debemos tratar de desarrollar ambas partes de manera integrada. Entrenar al jugador y a la persona dentro y fuera del campo.

- El jugador pensante: esto es, que sea capaz de tomar decisiones en cada contexto en el que se encuentre valorando la situación y aplicando sus conocimientos.

Cabe destacar que los primeros trabajos en Psicología del Deporte en el Villarreal C.F se remontan a la temporada 2006-2007 con un psicólogo. Actualmente el departamento está formado por cinco psicólogos del deporte a tiempo completo y tres a tiempo parcial.
A finales de la temporada 2014-2015, en el Villarreal se redefinió por parte de los entrenadores, en consenso tras largas jornadas de debate, el tipo de jugador que el Club estaba buscando con el objetivo de marcar una dirección en el camino y de, a partir de ahí, organizar y dar sentido al proceso (acciones). El jugador que pretende formar el Villarreal C.F, el que se pretende modelar, es un jugador autónomo (persona responsable tanto de su proceso educativo como de su desarrollo deportivo y personal), que entienda el juego (capaz de tomar decisiones analizando el entorno cambiante e interaccionar con los compañeros y rivales) y tenga valores que le permitan ser una persona íntegra y comprometida con la sociedad que le rodea.

Debido al tipo de jugador que se quiere formar, se debe ser coherente con los contextos de aprendizaje que se le presenta (Olmedilla, Ortega, Ortín y Andreu, 2008). En este sentido, los técnicos son los responsables de, no solo de generar dichos contextos de aprendizaje, sino modificar su forma de relacionarse con los jugadores, para que éstos se desarrollen en la línea buscada. Básicamente, para obtener un jugador con las características enunciadas en el párrafo anterior, es necesario entrenar de una manera concreta que favorezca todos estos aspectos. De manera intuitiva, parece que un entorno donde no se fomente la creatividad, la posibilidad de cometer errores y una cierta libertad para tomar decisiones permita el desarrollo del jugador que se pretende. Para conseguir dicho cambio se valoró como oportuno, por un lado, empezar a generar espacios de debate y aprendizaje con los entrenadores, con el fin de que se viera la necesidad de cambiar el foco hacia el jugador, y por otro lado, proponer la técnica de establecimiento de objetivos para la mejora de cada jugador y como paso previo y necesario, la mejora de los técnicos.

El establecimiento de objetivos es una técnica que ha sido utilizada en diferentes ámbitos sociales y suele llevar asociado un aumento en la motivación y en el rendimiento de las personas (Ortín y Olmedilla, 2001). Buceta (1998) destaca que esta técnica se puede utilizar para controlar variables como la motivación de logro, la autoconfianza, el nivel de activación, la atención, la agresividad y la cohesión de equipo.

En un artículo de referencia, Locke y Lathan (1985) destacaban que algunas de las características a tener en cuenta a la hora de establecer objetivos son: 
- Los objetivos específicos y difíciles favorecen mayor rendimiento que los poco específicos o fáciles.

- Los objetivos a corto plazo facilitan conseguir los objetivos a largo plazo.

- Los objetivos afectan al rendimiento influyendo en el esfuerzo, la perseverancia y la dirección de la atención, así como motivando estrategias de desarrollo.

- El feedback sobre el proceso es necesario para que el establecimiento de objetivos funcione.

- El deportista debe aceptar los objetivos para que funcionen. En el mundo del deporte es frecuente la utilización de objetivos en las planificaciones deportivas (Ortín y Olmedilla, 2001). Estos autores indican que en algunas ocasiones se producen errores al elegir los objetivos, ya que no se realiza un análisis riguroso y realista de las capacidades del deportista, así como del contexto de competición, ritmo de entrenamientos, momento de la temporada u otros factores que puedan influir. Además, como el resto de técnicas psicológicas, el establecimiento de objetivos necesita un período de aprendizaje por parte de los entrenadores y jugadores para ser efectivo. Otro aspecto clave a tener en cuenta es la adecuación entre los objetivos individuales y los colectivos ya que ambos tipos deben ir en consonancia (Díaz-Morales y García-Naveira, 2001).

El objetivo de este artículo es explicar y compartir con el lector cómo se ha desarrollado el proceso de elección del tipo de jugador que quiere formar el Villarreal C.F. y los pasos que consecuentemente se han tomado para crear los entornos de aprendizaje necesarios para su desarrollo a través de la técnica en establecimiento de objetivos. El presente trabajo se enmarca en la labor realizada en 8 de los equipos de fútbol base del Club durante las temporadas 2014-2015 y 2015-2016. Participaron en el proyecto el Villarreal C (formado casi en su totalidad por jugadores sub 20), el Juvenil A (formado casi en su totalidad por jugadores sub 19), el Juvenil Roda (formado casi en su totalidad por jugadores sub 18) , el Juvenil B (formado casi en su totalidad por jugadores sub 17), el Cadete A (formado casi en su totalidad por jugadores sub 16), el Cadete Roda (formado casi en su totalidad por jugadores sub 15), el Infantil A (formado casi en su totalidad por jugadores sub 14) y el Infantil Roda (formado casi en su totalidad por jugadores sub 13).

\section{Experiencia Profesional}

En este apartado presentamos las 5 fases en las que se ha dividido el proceso de aplicación de las ideas expuestas en la introducción. Cada fase contiene actividades (talleres, debates, etc.) que han servido para, en primer lugar, la toma de decisiones sobre qué tipo de jugador se quería, y en segundo lugar, definir qué acciones realizar para conseguirlo. Todas estas actuaciones fueron diseñadas y dirigidas por el Departamento de Metodología y el Departamento de Psicología del club:

- Fase 1: ¿Qué es el juego y qué tipo de jugador queremos? El objetivo era poner en común las diferentes maneras de entender el fútbol y llegar a consensos al respecto.

- Fase 2: Establecimiento de objetivos de los entrenadores. La finalidad era que cada uno de los entrenadores tuviera sus propios objetivos de mejora en cualquier ámbito que considerasen adecuado, buscando sobre todo objetivos que les pudieran ayudar en su desempeño profesional. Por ejemplo aprender determinadas habilidades de comunicación para explicar mejor las tareas en los entrenamientos. De esta manera, los entrenadores podrían vivir en primera persona el proceso que debían llevar a cabo con sus jugadores en las fases posteriores.

- Fase 3: Programa piloto de establecimiento de objetivos con los jugadores. El fin era que establecieran objetivos de manera libre con sus jugadores sin más conocimiento previo que el propio proceso de establecimiento de objetivos que ellos habían vivido en la fase 2.

- Fase 4: Establecimiento de objetivos con todos los jugadores. Una vez repasado el programa piloto de la Fase 3, en la Fase 4 se debían establecer los objetivos con las correcciones y ajustes aprendidos en la Fase 3 . En el presente programa se limitó a establecer solo objetivos individuales para cada jugador.

- Fase 5: Evaluación del proceso. El objetivo es comprobar la satisfacción de entrenadores y jugadores respecto al proceso de establecimiento de objetivos.

\section{Procedimiento}

\section{Fase 1.¿Qué es el juego y qué tipo de jugador queremos?}

En la primera fase se sentaron las bases del proyecto. Se desarrolló un programa previo donde los entrenadores debatieron durante ocho sesiones de 1 hora y 30 minutos cada una.

En esas sesiones, se trataron los siguientes temas:

- Cómo entendían el juego, con el fin de unificar el método de entrenamiento.

- Qué tipo de jugador queríamos formar, con el fin de definir la formación del jugador (ver introducción).

- Cómo entendían el contexto de entrenamiento desde la perspectiva pedagógica, con el fin de definir el método enseñanza/aprendizaje. 
A continuación, detallamos las dinámicas que abarcan de septiembre a diciembre de 2014.

\section{Reuniones entrenadores.}

a) Comprensión del juego.

- Objetivo/s: Unificar el método de entrenamiento y los sistemas de juego.

- Duración: 4 sesiones de 1 hora y 30 minutos cada una realizadas en un período de dos meses.

- Ubicación: Sala multiusos, en la ciudad deportiva.

Materiales: Una sala multiusos con las sillas puestas en círculo, una pizarra, un proyector y un ordenador con materiales (cortes de vídeo) preparados por el Departamento de Metodología.

- Desarrollo: Debate y puntos comunes, dirección con método socrático (preguntas para abrir debate y extracción de conclusiones). El director de Metodología apoyado por los psicólogos dirigían el debate en el que se buscan sinergias y un modelo común de entender el juego. Una vez expuestos los puntos de vista y terminado el debate se extraen los aspectos e ideas comunes y se define una forma de entender el juego general para todos.

b) Elección de tipo de jugador.

- Objetivo/s:

- Definir las cualidades a potenciar de los jugadores.

- Unificar criterios sobre la formación del jugador.

- Duración: 2 sesiones de 1 hora y 30 minutos cada una.

- Ubicación: Sala multiusos, en la ciudad deportiva.

- Materiales: Una sala multiusos con las sillas puestas en círculo, una pizarra, un proyector y un ordenador con materiales (cortes de vídeo) preparados por el Departamento de Metodología.

- Desarrollo: Nuevamente, el director de Metodología apoyado por los psicólogos dirigían el debate para unificar criterios en cuanto a los atributos y cualidades de los jugadores definiendo así un 'jugador tipo'.

c) Cambio de foco, del equipo al jugador y del resultado a la tarea.

- Objetivo/s:

- Entender el contexto enseñanza/aprendizaje.

- Centrarse en el desarrollo del jugador.

- Focalizar en la tarea.

- Duración: 2 sesiones de 1 hora y 30 minutos cada una.

- Ubicación: Sala multiusos, en la ciudad deportiva.

- Materiales: Una sala multiusos con las sillas puestas en círculo, una pizarra, un proyector y un ordenador con materiales (cortes de vídeo) preparados por el Departamento de Metodología.

- Desarrollo: el objetivo fue, partiendo de la base de los puntos comunes extraídos en las reuniones anteriores, reflexionar sobre el contexto enseñanza/aprendizaje. El tema principal fue la utilidad que tiene el contexto de com- petición, así como la relevancia que ocupa el contexto de entrenamiento.

\section{Fase 2. Establecimiento de objetivos de los entrenadores}

Tras los talleres de la fase 1, se buscaba que los entrenadores experimentaran y se familiarizaran con la técnica de establecimiento de objetivos. Para ello se elaboró un taller, realizado entre enero y febrero de 2015, para grupos reducidos por etapa (4 grupos de 4 personas) en los cuales, con ayuda del Departamento de Psicología, los primeros entrenadores de cada equipo fueron protagonistas del proceso de establecer sus propios objetivos de mejora.

\section{Taller: establecimiento de objetivos propios.}

- Objetivo/s:

- Familiarizarse con la técnica.

- Experimentar el proceso desde el otro lado.

- Empatizar con el jugador.

- Duración: 4 sesiones de 1 hora y 30 minutos cada una.

- Ubicación: Departamento de Psicología, en la Ciudad Deportiva.

- Materiales: Una sala con una mesa redonda, una pizarra y materiales y fichas elaborados por el departamento de psicología.

- Desarrollo: Análisis, planteamiento y elección del objetivo, seguimiento, evaluación y/o reformulación de los objetivos escogidos por los técnicos para ellos mismos durante el periodo citado anteriormente.

Ejemplos:

- Mejorar a nivel de conocimientos tácticos.

- Delegar más en el cuerpo técnico.

Como se puede apreciar, son objetivos mejorables a nivel de concreción, definición, operatividad, etc. Nuestra intención no era tanto que el objetivo estuviera definido perfectamente si no que 'sufrieran' las dificultades para conseguir cumplir una meta si no se tiene en cuenta el grado de control, que sea especifico, que sea medible, que dependa de uno mismo, que esté definido en el tiempo, que tenga un plan de acción y el resto de características deseables en un objetivo.

\section{Fase 3. Programa piloto de establecimiento de objetivos con los jugadores}

Tras estos talleres y con la experiencia de haber establecido sus propios objetivos, se pidió a los entrenadores que establecieran objetivos para sus jugadores a modo de experiencia piloto durante los meses de marzo y abril de 2015 con 
el doble fin de que los jugadores mejoraran su rendimiento y los entrenadores pudieran practicar con la técnica. Hay que destacar que los entrenadores no habían recibido materiales teóricos sobre la técnica de establecimiento de objetivos. Solo poseían la experiencia de los objetivos propios que se había establecido con ellos. La razón de este modo de actuación, sin la exposición previa a los fundamentos teóricos, fue por un lado ver qué conocimientos previos tenían los entrenadores, así como, darles la libertad para escoger cualquier objetivo que ellos quisieran. Era esperable que algunos objetivos no se ajustaran a los fundamentos teóricos enunciados en la introducción y que por tanto presentaran problemas a la hora de llevarlos a término. El no conocimiento de la técnica podía favorecer la experimentación y el descubrimiento de la idoneidad o desacierto de determinados objetivos. Por tanto, los entrenadores podían aprender de sus errores. A continuación, se muestran algunos ejemplos de objetivos que se plantearon. Como se puede apreciar también, son objetivos 'mejorables'. En este trabajo no vamos a entrar a discutir cómo se deberían ajustar dichos objetivos. Simplemente se ilustra el resultado del proceso por el que los entrenadores iban conociendo la técnica.

Una vez finalizado el plazo para preparar y probar los objetivos, se procedió a compartir las experiencias vividas, el método y técnicas utilizadas (como se ha explicado, tuvieron libertad de planteamientos aún sabiendo que podrían ser poco precisos o inadecuados). Se buscaba generar sinergias grupales, identidad colectiva y a su vez, estructurar un método común de establecimiento de objetivos entre todos, dónde se sintieran partícipes del mismo.

Además, se esperaba constatar que los técnicos pudieran tener sensaciones de acercamiento con sus jugadores además de sentir empatía, lo que podría derivar en mejora de las relaciones personales y aumento del conocimiento individual de sus jugadores independientemente de la adecuación del objetivo.

Después de establecer los objetivos con sus jugadores, los técnicos de los diferentes equipos prepararon una exposición breve en la que explicaron su experiencia con uno de los jugadores de su equipo para todos los entrenadores, coordinadores y psicólogos. En las presentaciones que se realizaron entre abril y mayo de 2015, los entrenadores plantearon sus iniciativas y valoraron qué aspectos habían funcionado bien y en qué aspectos habían tenido problemas. Mediante la colaboración y la puesta en común se fueron exponiendo los fundamentos de la técnica, utilizando los propios ejemplos expuestos por los entrenadores.

Ejemplos de los objetivos establecidos:

- El regate cerca de la portería rival.

- Debe ser capaz de mejorar el análisis del contexto en mo- mento con balón con la intención de progresar en el juego y obtener ventaja.

- Aunque ha mejorado notablemente en este aspecto, la comunicación con sus compañeros debe ser más fluida y espontánea.

Como en los ejemplos de los objetivos de la Fase 2, estos objetivos muestran evidentes aspectos de mejora en cuanto a definición. Todas estas posibilidades de mejora fueron comentadas tanto por los mismos técnicos como por los miembros del Departamento de Psicología y Departamento de Metodología, aprovechando la experiencia y las dificultades experimentadas por los entrenadores para entender y mejorar la aplicación de la técnica.

La Fase 3 terminó en la temporada 2014-2015 y se tenía el contexto perfecto para comenzar la temporada 2015-2016 y establecer los objetivos para todos los jugadores del club.

\section{Fase 4. Establecimiento de objetivos con todos los jugadores}

Se elaboró un taller en pretemporada (septiembre 2015) con el fin de consolidar la técnica así como los criterios y los puntos comunes generados la temporada anterior.

Taller: Establecimiento de objetivos en Villarreal CF.SAD.

- Objetivo/s:

- Consolidar y clarificar el método de establecimiento de objetivos.

- Consolidar los conocimientos teóricos básicos sobre dicha técnica.

- Enfatizar en los puntos comunes.

- Desarrollar con los entrenadores de un método común para iniciar el trabajo.

- Duración: 4 sesiones de 1 hora y 30 minutos cada una.

- Ubicación: Sala multiusos, en la ciudad deportiva.

- Materiales: Una sala multiusos con las sillas puestas en círculo, una pizarra, un proyector y un ordenador con materiales (cortes de vídeo) preparados por el Departamento de Metodología. El material teórico estaba basado en el capítulo de establecimiento de objetivos del libro de Fundamentos de Psicología del Deporte de Weinberg y Gould (Weinberg y Gould, 2007).

- Desarrollo: Reuniones dirigidas mediante método socrático y debate abierto por el Departamento de Psicología y Departamento de Metodología. Partiendo de los ejemplos expuestos, se hizo un trabajo de descubrimiento de los fundamentos de la técnica que detallamos a continuación:

- Entrega a los entrenadores de material teórico con los fundamentos de la técnica.

- Tipos de objetivos. 
- Reunión del Cuerpo Técnico para realizar el análisis del jugador partiendo de un período de observación en entrenamientos y partidos durante la pretemporada (agosto 2015).

- Entrevista con el jugador para establecer los objetivos.

- Desarrollo del plan de acción con el jugador.

- Evaluación del objetivo.

A lo largo de la temporada, se realizaron sesiones de seguimiento con el formato citado anteriormente (debates y exposición de casos prácticos), se exponían el análisis del jugador por parte de los cuerpos técnicos, así como los diferentes objetivos, planes de acción y modo de evaluación. Mediante una metodología colaborativa, se debatía sobre si los objetivos se ajustaban a los criterios expuestos anteriormente.

Ejemplo de objetivos de la temporada 2015-2016.

- Ofrecer soluciones a los compañeros en posesión del balón.

- Mayor contundencia en las disputas en balones aéreos.

- Asociarse con sus compañeros cuando está en posesión del balón, ya que normalmente quiere hacer jugada individual.

Los objetivos del ejemplo cumplen algunas de las características deseables. Empiezan a aparecer los verbos en infinitivo y aparecen las conductas que dependen solo del jugador. Aun así, siguen siendo objetivos mejorables en cuanto a su definición y son estos aspectos los que se fueron trabajando en las sesiones de seguimiento.

El proceso concluye con el cierre de la temporada y su posterior informe final en junio de 2016. En dicho informe, de carácter privado y confidencial, figura todo el proceso trabajado con el jugador durante el año. Para ello, siguiendo el sistema que se ha utilizado durante todo el proceso, se realizó una dinámica en marzo 2016 donde se definió qué modelo de informe se debía realizar.

\section{Dinámica: Modelo de informe.}

- Objetivo/s:

- Establecer un modelo de informe común.

- Analizar los factores a los que hay que darles relevancia.

- Facilitar el proceso de conocimiento del jugador a los siguientes cuerpos técnicos.

- Duración: 1 sesión de 1 hora y 30 minutos cada una.

- Ubicación: Sala multiusos, en la ciudad deportiva.

- Materiales: Una sala multiusos con las sillas puestas en círculo, una pizarra, un proyector y un ordenador con materiales (Power Point con modelos de informe tipo realizado por los diferentes grupo de trabajo de cada etapa).

- Desarrollo: Después de cada presentación, se debatió sobre los diferentes modelos y formatos eligiéndose un modelo de informe en Power Point al que fueron añadidas diferentes características interesantes de otros modelos.

\section{Fase 5. Evaluación del proceso}

Como parte final, se ha realizó una evaluación a los jugadores y técnicos participantes (García-Naveira, 2017; Olme-

Tabla 1. Resultados descriptivos (Media y Desviación típica) de la valoración del empleo de la técnica de establecimiento de objetivos por parte de los entrenadores $(n=77)$ y jugadores $(n=169)$

\begin{tabular}{llllllllllll}
\hline Equipo & IR & IA & CR & CA & JB & JR & JA & VC & CT & Total
\end{tabular}

Ítem 1: ¿Te motiva trabajar con establecimiento de objetivos?

\begin{tabular}{ccccccccccc}
$\overline{\mathrm{X}}$ & 4.3 & 3.7 & 4 & 3.8 & 3.8 & 3.7 & 4.2 & 4.3 & 4.5 & 4 \\
\hline$\sigma$ & 1 & 1 & 1 & 1 & 1 & 0.75 & 0.8 & 0.7 & 0.7 & 0.9
\end{tabular}

Ítem 2: ¿Te resulta útil trabajar con establecimiento de objetivos?

\begin{tabular}{ccccccccccc}
$\overline{\mathrm{X}}$ & 4.6 & 3.9 & 3.9 & 3.8 & 4 & 3.9 & 4.2 & 4.2 & 4.5 & 4.1 \\
\hline$\sigma$ & 0.6 & 0.9 & 1 & 0.9 & 0.9 & 0.8 & 0.7 & 0.7 & 0.6 & 0.8
\end{tabular}

Ítem 3: ¿Te gustaría continuar con la técnica de establecimiento de objetivos la temporada siguiente?

\begin{tabular}{ccccccccccc}
$\overline{\mathrm{X}}$ & 4.4 & 3.4 & 4.1 & 3.7 & 4 & 3.9 & 4.4 & 3.8 & 4.5 & 4 \\
\hline$\sigma$ & 1 & 1.4 & 1.2 & 1 & 1 & 0.7 & 0.8 & 0.9 & 0.6 & 1
\end{tabular}

Nota IR = Infantil Roda de primer año (sub13); IA = Infantil A de segundo año (sub14); CR = Cadete Roda de primer año (sub15); CA = Cadete A de segundo año (sub16); JB = Juvenil B de primer año (sub17); JR = Juvenil Roda de segundo año (sub18); JA = Juvenil A de tercer año (sub19); VC = Villarreal C (sub20); CT = Cuerpo Técnico. 
dilla y Dominguez-Igual, 2016). La evaluación se ha realizó con un breve cuestionario ad hoc donde se medía mediante una escala tipo likert de 1 a 5 puntos el grado de satisfacción con la técnica, la utilidad percibida así como la intención de continuar con la técnica en temporadas siguientes. El cuestionario también tenía una pregunta abierta para que pudieran expresar libremente su opinión. A continuación se presenta una breve descripción de los resultados obtenidos.

El cuestionario fue elaborado con la herramienta de crear cuestionarios de Google Drive y se envió a los participantes un enlace para participar vía mensaje de texto al teléfono móvil. El cuestionario era totalmente anónimo.

En la evaluación participaron 77 técnicos con una media de edad de 29.7 años $(D T=6.3)$ y 169 jugadores con una media de edad de 16.4 años $(D T=2.3)$, repartidos en las diferentes categorías deportivas establecidas con anterioridad (ver Tabla 1).

En cuanto a los resultados cuantitativos solo se comentaran las puntuaciones totales. Los cuerpos técnicos han valorado muy positivamente (puntuación de 4.5 sobre 5) la motivación para trabajar con dicha técnica, la utilidad así como la intención de seguir utilizando la técnica. Por lo que respecta a los equipos, teniendo en cuenta que la puntuación máxima era 5 , los equipo con puntuaciones más bajas han sido el Infantil A y el Cadete A, aun siendo puntuaciones por encima de 3 . El resto de los equipos muestran puntuaciones parecidas a las obtenidas por los técnicos en cuanto a motivación, utilidad de la técnica y deseo de continuar con su práctica.

Además de los ítems valorados, disponía una opción de respuesta cualitativa "¿Qué te ha aportado trabajar este año con objetivos individuales?". Se decidió añadir este tipo de pregunta para darles la oportunidad de profundizar en su experiencia. Definidas por dos psicólogos del club, las respuestas se han agrupado en categorías atendiendo al aspecto al que se referían. De todas ellas, el aspecto más destacado por los jugadores ha sido el de mejora personal/deportiva que incluye respuestas del tipo "mejorar en detalles para ser mejor cada día", "me ha ayudado a mejorar las cosas que no sabía mejorar bien", "para ver en qué soy más flojo en el campo y mejorarlo para mi bien y para el del equipo", "poder mejorar yo mismo y valorar más cosas en el juego" o "me ha venido muy bien porque son cosas en las que te ayuda para tu carrera como futbolista". La segunda categoría más destacada por los jugadores ha sido la de cambios en su capacidad de atención/pensamientos. Esta categoría incluye respuestas como: "Me ha ayudado mucho en la forma de jugar y he sacado ventaja en los partidos y entrenamientos y también en los pensamientos que tenía antes y tengo ahora", "enfocarme más en estos objetivos para mejorar", "con- centrar en mejorarlos", "fijarme en cosas que antes no me fijaba", "el estar muy pendiente de ese objetivo y olvidarme de cosas externas negativas como por ejemplo: si algo no me va bien en mi vida, los estudios... Antes a lo mejor iba a entrenar y traía esos pensamientos pero ahora intento sólo pensar en mí objetivo y olvidarme de lo demás", "prestar más atención a situaciones que no le daba importancia y me han ayudado a mejorar", "fijarme en lo que fallaba y al poner los objetivos he intentado mejorarlo para cada vez fallar en menos cosas y mejorar como jugador". Una tercera categoría hace referencia al aprendizaje y engloba respuestas del estilo: "El año pasado me ayudó y este año he podido aprender nuevas cosas", "verme en vídeos es muy importante para la hora de jugar en el campo puedo ver los errores, lo que hago bien", "he podido corregir muchos errores de forma más rápida" o "convertir mis errores en el campo en cosas buenas".

En cuanto a los entrenadores, los aspectos más valorados se han agrupado en categorías que hacen referencia a aspectos como cercanía con el jugador con respuestas del tipo: "conocer más de los jugadores y sus inquietudes", "conocer mejor a los chavales, ya que interactúas más con ellos y hay más charlas. Mayor confianza con ellos.", "la cercanía con los jugadores nos ha dado la posibilidad de que la relación jugador-entrenador sea mucho más cercana, también hemos notado que los jugadores han mejorado significativamente desde el inicio de temporada", "una relación más eficaz y cercana al jugador que me permite trabajar de forma conjunta con él por una mejora en juego", "una nueva forma de intentar llegar al niño e intentar mejorar los errores que puede cometer, o potenciar sus cualidades. Además de que a él le resulta bastante útil para ver lo que está realizando y cómo mejorarlo por sí solo" o "todo este trabajo me ha permitido conocer y empatizar mucho más a los jugadores con lo que la ayuda prestada es mucho mayor o más precisa sintiéndose el jugador escuchado y ayudado". Otra categoría destacada hace referencia a la propia mejora del entrenador: "una mayor capacidad de análisis respecto a los jugadores y al juego. También me ha aportado el crecimiento, en general, como entrenador al tener que utilizar diferentes herramientas para poder llevar a cabo dicho análisis global de cada jugador", "me ha aportado tener que ser mucho más cauteloso y fijarme en muchas cosas más de las que hasta día de hoy no hacía", "aumentar mis conocimientos y mis recursos futbolísticos", "ha hecho plantearme retos y motivaciones a conseguir que los jugadores tengan una mejora continua en diferentes contextos" o "me ha aportado el intentar comprender mucho más el juego del equipo para así poder extraer los objetivos individuales propios. Además, el hecho de establecer objetivos te hace ser más constante en tu trabajo y dedicación hacia él”. 


\section{Discusión}

Como describen Ortin y Olmedilla (2001), los deportistas suelen estar acostumbrados a asistir a los entrenamientos, escuchar y ejecutar las instrucciones del entrenador y marcharse a casa a esperar al día siguiente. Se comporta como un sujeto relativamente pasivo que reacciona y ejecuta las instrucciones que llegan de fuera. Muchos entrenadores piden jugadores creativos y con iniciativa. Normalmente, se les entrena y refuerza sin darles mucha opción de que tengan iniciativas en cuanto a su mejora ni tampoco, voz ni voto en el trabajo que deben realizar en su proceso de aprendizaje.

El trabajo presentado es un proceso en desarrollo. Se ha tratado de explicar el procedimiento para crear unas bases sólidas en cuanto a qué tipo de jugador se quería desarrollar y qué estrategias se iban a utilizar para conseguirlo. La división en las fases explicadas corresponde a las necesidades del Club de definir un modelo de jugador común y un método para conseguirlo. A continuación, exponemos los puntos destacables a nuestro entender:

- Fomento de la participación: se ha utilizado una metodología eminentemente participativa para 'elegir' el tipo de jugador a desarrollar. De esta manera se consigue que el objetivo sea 'de todos' y no impuesto explícitamente desde la dirección deportiva.

- Aprendizaje por descubrimiento: durante la mayoría del proceso, los entrenadores y jugadores han tenido que 'descubrir' cómo se establecen los objetivos y qué dificultades y soluciones se pueden utilizar al respecto. Entendemos que este es un aspecto novedoso ya que cuando se implementa este tipo de técnicas, se suele instruir de manera sistemática a los técnicos y jugadores en dicho procedimiento en lugar de guiarlos para que lo descubran por ellos mismos como se ha hecho en este caso. De esta manera, se fomentaba la creatividad y se podía experimentar el hecho de cometer errores y poder aprender de ellos.

- Sistematización del proceso de aprendizaje: el hecho de tener que analizar a un jugador, establecer con él unos objetivos de mejora, crear un plan de acción para conseguirlo y evaluar el grado de consecución del objetivo permite que el desarrollo del jugador se pueda seguir y continuar de una temporada a otra y dar continuidad al trabajo con dicho jugador. En el mundo del fútbol de iniciación, es muy común que cada equipo trabaje con las directrices de cada entrenador concreto durante una temporada concreta. De esta manera, es muy difícil poder encontrar información del trabajo previo realizado con un jugador en temporadas anteriores y es muy difícil hacer un seguimiento más o menos objetivo tanto del desarrollo como de las estrategias utilizadas. Al tener toda la información sobre el proceso con el jugador registrado, se podrá seguir con mayor detalle el currículo deportivo de cada jugador.

Al ser el presente trabajo una explicación de un proceso aplicado, se pueden encontrar limitaciones y licencias, sobre todo nivel metodológico propias del desempeño del día a día en un entorno sometido al cambio continuo como es el fútbol. No obstante, la voluntad ha sido la de compartir y explicar la experiencia.

Como conclusión, es cierto que se podrían haber utilizado otras maneras de explicar desde el principio como se establecen los objetivos (directivo). En nuestro caso, se ha optado por un proceso en el que el aprendizaje de la técnica es vivencial, con el fin de que se den cuenta de las dificultades con las que se pueden encontrar cuando los objetivos son difusos y poco concretos; que se den cuenta de lo difícil que es comunicarse con los jugadores; que valoren la importancia y sientan los objetivos como suyos; que el hecho de hablar con el jugador les haga replantearse sus habilidades como comunicadores; que les haga salir de la zona de confort en la que también se encuentran los técnicos entrenando 'como siempre'; que les obligue a entender el error como una parte principal del proceso. Resulta difícil dejar 'fallar' al jugador o verlo dar respuestas que no son óptimas o las que se esperan y aun así, facilitar que el jugador pueda decidir qué hacer para mejorar el resultado de sus decisiones o acciones por sí mismo.

Nuestra intención es seguir trabajando en el proceso de aprendizaje que hemos iniciado e ir extrayendo conocimientos y experiencias que sean aplicables y transferibles a otros conceptos y situaciones. En posteriores trabajos, se presentarán ejemplos de objetivos establecidos en las determinadas etapas, los planes de acción para conseguir esos objetivos así como la manera de evaluarlos.

Aspectos interesantes para futuros trabajos podría ser:

- Autogestión de los objetivos por parte de los jugadores.

- Trabajo de objetivos comunes para dos o más jugadores y el equipo.

- Supervisión del proceso de mejora por parte de otros jugadores y equipos del Club.

- Trabajar sobre los aspectos más fuertes del jugador para potenciarlos aun más.

\section{Referencias}

Buceta, J. M. (1998). Psicología del Entrenamiento Deportivo. Madrid, España: Dykinson.

Díaz-Morales, J. F. y García-Naveira, A. (2001). Evaluación de metas en jugadores de fútbol de categoría juvenil y absoluta. Revista de Psicología del Deporte, 10(2), 211-223. 
García-Naveira, A. (2017). Entrenamiento psicológico para la mejora del autocontrol en un entrenador de fútbol. Acción Psicológica, 14(1), 27-42. https://doi.org/10.5944/ap.14.1.19255

García-Naveira, A. y Jerez, P. (2012). Departamento de psicología del club Atlético de Madrid: filosofía, programación y desempeño profesional en el fútbol base. Cuadernos de Psicología del Deporte, 12(1), 111-120. https://doi.org/10.4321/S157884232012000100010

Morilla, M., Pérez, E., Gamito, J., Gómez, M., Sánchez, J. y Valiente, M. (2003).Planificación psicológica de la cantera del Sevilla F.C. S.A.D.: organización, funcionamiento y programa deportivo-formativo. Cuadernos de Psicología del Deporte, 3(2), 17-30.

Locke, E. A., Shaw, K. M., Saari, L. M. y Latham, G. P. (1981). Goal setting and taskperformance: 1969-1980. Psychological Bulleting, 90, 125-152. https://doi.org/10.1037/0033-2909.90.1.125

Locke, J. y Lathan, G. P. (1985) The application of goal setting to sports. Journal of Sport Psychology, 7, 205-222. https://doi. org/10.1123/jsp.7.3.205

Olmedilla, A. y Dominguez-Igual, J. (2016). Entrenamiento psicológico para la mejora de la atención y la autoconfianza en un futbolista. Revista de Psicología Aplicada al Deporte y al Ejercicio Físico, 1, e4. http://dx. doi.org/10.5093/rpadef2016a4

Olmedilla, A., Ortega, E., Ortín, F. y Andreu, M.D. (2008). Entrenamiento psicológico en fútbol base de élite: percepción de aplicabilidad e índices de satisfacción. Revista de Iberoamericana de Psicología del Ejercicio y el Deporte, 3(1), 31-46.

Ortín, F. J. y Olmedilla, A. (2001). El establecimiento de objetivos como herramienta para la mejora del rendimiento en deportes de equipo. Cuadernos de Psicología del Deporte, 1(1), 91-100.

Weinberg, R. y Gould, D. (2007). Foundations of Sport and Exercise Psychology. Champaign, II, Estados Unidos: Human Kinetics.
ANEXO: Items de evaluación de la técnica en establecimiento de objetivos

a) ¿Te motiva trabajar con objetivos? (escala Likert 1 (mínimo)-5 (máximo))

b) ¿Te resulta útil trabajar con objetivos? (escala Likert 1 (mínimo)-5 (máximo))

c) ¿Te gustaría continuar con esta técnica de objetivos la temporada siguiente? (escala Likert 1 (mínimo)-5 (máximo))

d) ¿Qué te ha aportado diferente trabajar este año con objetivos individuales? (respuesta de texto). 